\title{
Association of TCF7L2 and ADIPOQ with Body Mass Index, Waist-Hip Ratio, and Systolic Blood Pressure in an Endogamous Ethnic Group of India
}

\author{
Vipin Gupta, Rajesh Khadgawat, ${ }^{2}$ H. K. Tony Ng, ${ }^{3}$ Gagandeep Kaur Walia, Leena Kalla, \\ Vadlamudi Raghavendra Rao, ${ }^{4}$ and Mohinder Pal Sachdeva ${ }^{4}$
}

Despite the increasing burden of type 2 diabetes (T2D) and its established association with anthropometric and physiological traits as a risk factor, genetic studies focusing on the association of T2D-related genes with quantitative traits like body mass index (BMI), waist-hip ratio (WHR), and systolic blood pressure (SBP) are only a few for western populations and rare for Indian populations. The present study tested the association of TCF7L2, HHEX, KCNJ11, and ADIPOQ with BMI, SBP, and WHR in men and women of the Aggarwal population of India and found a differential association of TCF7L2 (rs7903146, rs4506565, and rs12256372) and ADIPOQ (rs2241766 and rs1501299) genes with increasing BMI, SBP, and WHR between the two sexes. We conclude that TCF7L2 and ADIPOQ together might play an important role in explaining these traits and to understand the biological and genetic mechanisms underlying T2D, and the role of other T2D genes must also be evaluated with these continuous traits.

\section{Introduction}

$\mathrm{O}$ BESITY AND DIABETES in India have already achieved a numero uno status, and its epidemic is expected to rise further in the near future (International Diabetes Federation Report, 2009). Association of increase in various anthropometric (e.g., body mass index [BMI] and waist-hip ratio [WHR]) and physiological (e.g., systolic blood pressure [SBP]) measures with obesity and diabetes is well established (Vazquez et al., 2007; Mullican et al., 2009).

The advent of genome-wide association studies (GWAS) uncovered many genetic variants related to obesity and type 2 diabetes (T2D) and led to an ongoing series of efforts for their validation in different human populations (Horikoshi et al., 2007). The lack of GWAS from India due to absence of large consortiums and only limited infrastructure along with small funding are forcing Indian genetic epidemiologists to produce at least validation studies of GWAS genes (e.g., Chauhan et al., 2010). Despite of our knowledge that obesity is a risk factor for diabetes, very few genetic studies have tested the hypothesis that the genes related to diabetes are also associated with obesity-related continuous traits (e.g., BMI, WHR, and SBP) by significantly increasing their mean value (Helgason et al., 2007; Pecioska et al., 2010). Sanghera et al. (2008) studied the impact of T2D genes on BMI, WHR, and lipid profile, but only found the association of TCF7L2 with the cholesterol level in an Indian population. Therefore, in this article, we are presenting the results of testing the above hypothesis by evaluating the association of diabetes genes with anthropometric and physiological measures. We have found that T2D genes might play a role in explaining the genetics of anthropometric and physiological risk factors in India.

\section{Materials and Methods}

This article is a part of the case-control genetic association study for T2D among the Aggarwals of Delhi; the research design is given in Gupta et al. (2010). This endogamous population (intermarriage between clans) group was selected primarily to avoid false-positive association signals due to population stratification, secondly, due to its large population size (2-3 million in Delhi and surrounding area), and finally because this is a traders' community having a sedentary life style. Fortunately, this endogamous genetic structure of the Indian population has been comprehensively proved by the landmark study of Reich et al. (2009). For the purpose of analysis, we had first grouped the available case-control data (300 samples for which data were available from the parent

\footnotetext{
${ }^{1}$ South Asia Network for Chronic Disease (SANCD), Public Health Foundation of India, New Delhi, India.

${ }^{2}$ Department of Endocrinology and Metabolism, All India Institute of Medical Sciences, New Delhi, India.

${ }^{3}$ Department of Statistical Science, Southern Methodist University, Dallas, Texas.

${ }^{4}$ Department of Anthropology, University of Delhi, Delhi, India.
} 
study) for our target of continuous traits (BMI, WHR, and $\mathrm{SBP}$ ), and then all data were segregated on the basis of the sex. The study was ethically cleared by the All India Institute of Medical Sciences, New Delhi.

All the genotyped 14 single-nucleotide polymorphisms (SNPS; TCF7L2-3 SNPs, HHEX - 2 SNPs, KCNJ11-1 SNP, and $A D I P O Q-8 \mathrm{SNPs}$ ) generated through sequencing (see Supplementary Data available online at www.liebertonline .com/gtmb) were used for the analysis. For testing the association of SNPs along with six variables (sex, age, WHR, physical activity, socioeconomic status, and SBP), the dataset was first cleaned by removing the observations with missing values $>4$ out of the 6 variables; therefore, several observations (111 for BMI, 94 for SBP, and 120 for WHR) were removed, and then the R-package-"Hmisc" (Harrell, 2010) was used for imputations of the missing values of variables. Multiple regression models were fitted for all the SNPs, and then based on a step-wise selection, using the Akaike's information criterion, potential SNPs were identified separately for BMI, WHR, and SBP. Thereafter, multiple regression models were fitted for a single-SNP to detect association. All the analyses were done using $\mathrm{R}$ ( $\mathrm{R}$ Development Core Team, 2008).

\section{Results}

All the 14 SNPs in four genes (TCF7L2, HHEX, KCNJ11, and $A D I P O Q)$ were found to be in Hardy-Weinberg equilibrium (Supplementary Table S1). While testing for associations with continuous traits like BMI, SBP, and WHR, only two genes (TCF7L2 and ADIPOQ) were found to be significant. The number of men and women tested for association is given in Supplementary Table S1. For BMI, we found two significant SNPS of TCF7L2, that is, rs4506565 (genotype-AT increasing BMI to 27.37, $p=0.009$ ) and rs7903146 (genotype-CT increasing BMI to 27.45, $p=0.019)$ among men, and one SNP of $A D I P O Q$, that is, rs2241766 (genotype-AT increasing BMI to 34.24, $p=0.048$ ) among women (Table 1). Similarly, for SBP, we found same two significant SNPs of TCF7L2, that is, rs4506565 (genotype-TT increasing SBP to $141.35 \mathrm{mmHg}$, $p=0.01$ ) and rs7903146 (genotype-TT increasing SBP to 141.12, $p=0.028$ ) among men, and one SNP of ADIPOQ, that is, rs2241766 (genotype-AT decreasing SBP to 124.78, $p=0.042$ ) among women (Table 1). For WHR, we found one SNP of ADIPOQ, that is, rs1501299 (genotype-GT increasing WHR to $1.026, p=0.02$ ) among men, and one significant SNP of TCF7L2, that is, rs12256372 (genotype-GT increasing WHR to $0.921, p=0.029$ ) among women (Table 1). When we treated one of the alleles having lower frequency as the risk allele, the number of categories reduced from three to two by combining it into two categories (based on the risk and nonrisk category). While testing in this two-category scenario, except for BMI in men, we were unable to detect significant association for other target quantitative variables. For BMI, we found TCF7L2 SNP rs4506565 (AT + TT risk category) significantly increasing BMI to 27.15 among men.

\section{Discussion}

Despite of the discovery of loci such as TCF7L2, HHEX, $F T O$, and CDKN2B, through GWAS, where cases were not selected for BMI (Scott et al., 2007), the role of T2D genes has not been much evaluated for risk factors like BMI, WHR,
Table 1. Effect of Single-Nucleotide Polymorphisms of TCF7L2 ANd ADIPOQ on Adjusted Body Mass Index and Systolic Blood Pressure and Waist-Hip Ratio

\begin{tabular}{|c|c|c|c|c|c|}
\hline Gene & SNP & Genotype & $\begin{array}{l}\text { Trait } \\
\text { Value }\end{array}$ & $\begin{array}{l}\text { Regression } \\
\text { coefficient }\end{array}$ & $\mathrm{p}$ \\
\hline \multicolumn{6}{|l|}{ BMI: men } \\
\hline \multirow[t]{3}{*}{ TCF7L2 } & rs 4506565 & AA & 25.48 & & \\
\hline & & $\mathrm{AT}$ & 27.37 & 0.0716 & 0.009 \\
\hline & & TT & 26.52 & 0.0402 & 0.285 \\
\hline \multirow[t]{3}{*}{ TCF7L2 } & rs7903146 & $\mathrm{CC}$ & 25.77 & & \\
\hline & & $\mathrm{CT}$ & 27.45 & 0.063 & 0.019 \\
\hline & & $\mathrm{TT}$ & 25.90 & 0.005 & 0.885 \\
\hline \multicolumn{6}{|c|}{ BMI: women } \\
\hline \multirow[t]{3}{*}{$A D I O P Q$} & rs2241766 & TT & 29.08 & & \\
\hline & & TG & 30.02 & 0.031 & 0.452 \\
\hline & & GG & 34.24 & 0.163 & 0.048 \\
\hline \multicolumn{6}{|c|}{ SBP: men } \\
\hline \multirow[t]{3}{*}{ TCF7L2 } & rs4506565 & $\mathrm{AA}$ & 132.34 & & \\
\hline & & $\mathrm{AT}$ & 130.62 & -0.005 & 0.806 \\
\hline & & TT & 141.35 & 0.077 & 0.010 \\
\hline \multirow[t]{3}{*}{ TCF7L2 } & rs7903146 & $\mathrm{CC}$ & 132.51 & & \\
\hline & & $\mathrm{CT}$ & 130.56 & -0.014 & 0.501 \\
\hline & & $\mathrm{TT}$ & 141.12 & 0.062 & 0.028 \\
\hline \multicolumn{6}{|c|}{ SBP: women } \\
\hline \multirow[t]{3}{*}{$A D I O P Q$} & rs2241766 & TT & 132.99 & & \\
\hline & & TG & 124.78 & -0.063 & 0.042 \\
\hline & & GG & 135.72 & 0.020 & 0.742 \\
\hline \multicolumn{6}{|c|}{ WHR: men } \\
\hline \multirow[t]{3}{*}{$A D I O P Q$} & rs1501299 & GG & 0.998 & & \\
\hline & & GT & 1.026 & 0.027 & 0.020 \\
\hline & & TT & 1.012 & 0.014 & 0.558 \\
\hline \multicolumn{6}{|c|}{ WHR: women } \\
\hline \multirow[t]{3}{*}{ TCF7L2 } & rs12256372 & GG & 0.887 & & \\
\hline & & GT & 0.921 & 0.037 & 0.029 \\
\hline & & TT & 0.887 & 0.0002 & 0.989 \\
\hline
\end{tabular}

For BMI, association is adjusted for age, WHR, PAS, SES, and SBP. For SBP, association is adjusted for age, WHR, PAS, SES, and BMI. For WHR, association is adjusted for age, SBP, PAS, SES, and BMI. SNP, single-nucleotide polymorphism; BMI, body mass index; SBP, systolic blood pressure (mmHg); WHR, waist-hip ratio; PAS, Physical Activity Score; SES, Socioeconomic Status Score.

and SBP. For instance, Pecioska et al. (2010) analyzed T2Dassociated risk alleles (related to nine genes) with human measures of fatness like the BMI, the fat mass index, fat percentage, waist circumference, and WHR and found association only with the FTO gene, a well-established gene for obesity. However, not even a single study has evaluated the possibility for these associations in an Indian context. Therefore, we tried to detect associations between T2D SNPs and these diabetes-related continuous traits.

Since anthropometric traits, alone (height, weight, etc.) and in combination (like BMI and WHR), have different standards for men and women and the variation corresponding to these differences influences the risk for T2D for them, we therefore analyzed the effect of SNPs on these continuous traits separately. We found the significant association of TCF7L2 SNPs like rs4506565 (AT) and rs7903146 (CT) among men and AQIPOQ SNP rs2241766 (GG) among women with an increase in the mean BMI of this population. One cross-sectional 
study has shown that with increasing BMI and decreasing adiponectin levels in girls could contribute to their faster deterioration in lipid profiles in comparison with boys (Ong et al., 2006). To some extent, this explains the significant association of $A D I P O Q$ with BMI among women.

Despite a well-appreciated validation of TCF7L2 SNPs in different world populations, the association of TCF7L2 with obesity-related quantitative traits and body fat in humans is still unclear (Haupt et al., 2010). For TCF7L2, we found a heterozygous genotype significant for BMI but not the homozygous genotype, possibly due to lack of enough power to capture its effect or maybe because TCF7L2 has effect on increasing BMI in its heterozygous form. The role of ADIPOQ is known for obesity, but its differential role in men and women remains yet to be evaluated. Among women, homozygous rs2241766-GG (ADIPOQ) has the effect of increasing BMI, which may be due to a recessive effect on BMI.

Similarly, for SBP, we found a significant effect of TCF7L2 SNPs (like rs4506565-TT and rs7903146-TT) for men and ADIPOQ SNP rs2241766-TG among women in increasing in the mean SBP. For SBP, this study has sufficient power to capture a homozygous effect of TCF7L2 SNPs, which is likely to have a recessive effect on SBP.

Both TCF7L2 and ADIPOQ are expressed in adipose tissue and involved in Wnt-dependent regulation of adipogenesis (Ross et al., 2002; Sladek et al., 2007; Cauchi et al., 2008), which maintains pre-adipocytes in an undifferentiated state (Ross et al., 2002). The few studies that analyzed the effect of TCF7L2 SNPs on BMI (Schafer et al., 2007; Grant et al., 2006; Zhang et al., 2006; Helgason et al., 2007) hinted toward the possible association, but were not confirmed. Haupt et al. (2010) investigated the association of TCF7L2 variants with body fat composition and ectopic lipid storage in a cross-sectional setting and found that the TCF7L2 gene influenced lifestyle intervention-induced changes in BMI, total fat, and nonvisceral and visceral fat, but these results must be replicated as suggested by the authors.

Interestingly, for WHR we found a significant effect of ADIPOQ SNP (rs1501299-GT) for men and TCF7L2 SNP (rs12256372-GT) among women in increasing the mean WHR. Since both men and women have a different body composition, it is expected that the genes explaining the BMI and WHR might also differ, but the present study is not sufficient to support this hypothesis.

Thus, in our study also, both TCF7L2 and ADIPOQ are found to be associated with BMI, SBP, and WHR and point in the same direction; this supports the growing evidence for the hypothesized role of TCF7L2 in adipocyte differentiation (Haupt et al., 2010). The detected differences in genetic association in the present study among men and women need replication with a larger sample size to confirm these findings. It is difficult to say that the nonassociation of other SNPs of HHEX and KCNJ11 is because they do not have any effect on these continuous traits or due to low power.

We conclude that TCF7L2 and ADIPOQ together might play an important role in regulating the human BMI, WHR, and SBP in the Aggarwal population; therefore, to confirm this, their role must be evaluated through larger genetic and epigenetic studies. In the end, we would like to emphasize that to understand the underlying, overlapping biological pathways and genetic spectrum of T2D, the role of T2D genes must be evaluated with these continuous traits.

\section{Acknowledgment}

Maharaja Agresen Hospital, New Delhi; Anthropological Survey of India, Mysore; and the Aggarwal community. Funding Source: Anthropological Survey of India, Ministry of Culture, India and Indian Council of Medical Research, New Delhi.

\section{Author Disclosure Statement}

No competing financial interests exist.

\section{References}

Cauchi S, Choquet H, Gutierrez-Aguilar R, et al. (2008) Effects of TCF7L2 polymorphisms on obesity in European populations. Obesity 16:476-482.

Chauhan G, Spurgeon CJ, Tabassum R, et al. (2010) Impact of common variants of PPARG, KCNJ11, TCF7L2, SLC30A8, HHEX, CDKN2A, IGF2BP2 and CDKAL1 on the risk of type 2 diabetes in 5164 Indians. Diabetes 59:2068-2074.

Grant SF, Thorleifsson G, Reynisdottir I, et al. (2006) Variant of transcription factor 7-like 2 (TCF7L2) gene confers risk of type 2 diabetes. Nat Genet 38:320-323.

Gupta V, Khadgawat R, Ng HKT, et al. (2010) A validation study of type 2 diabetes-related variants of the TCF7L2, HHEX, KCNJ11, and ADIPOQ genes in one endogamous ethnic group of North India. Ann Hum Genet 74:361-368.

Harrell FE (2010) Hmisc: Harrell Miscellaneous. Available at http://CRAN.R-project.org $/$ package $=$ Hmisc

Haupt A, Thamer C, Heni M, et al. (2010) Gene variants of TCF7L2 influence weight loss and body composition during lifestyle intervention in a population at risk for type 2 diabetes. Diabetes 59:747-750.

Helgason A, Palsson S, Thorleifsson G, et al. (2007) Refining the impact of TCF7L2 gene variants on type 2 diabetes and adaptive evolution. Nat Genet 39:218-225.

Horikoshi M, Hara K, Ito C, et al. (2007) A genetic variation of the transcription factor 7-like 2 gene is associated with risk of type 2 diabetes in the Japanese population. Diabetologia 50: 747-751.

International Diabetes Federation Report (2009) Available at www.diabetesatlas.org.

Mullican DR, Lorenzo C, Haffner SM, (2009) Is prehypertension a risk factor for the development of type 2 diabetes? Diabetes Care 32:1870-1872.

Ong KK, Frystyk J, Flyvbjerg A, et al. (2006) Sex-discordant associations with adiponectin levels and lipid profiles in children. Diabetes 55:1337-1341.

Pecioska S, Zillikens MC, Henneman P, et al. (2010) Association between type 2 diabetes loci and measures of fatness. PLoS One 5:e8541.

R Development Core Team (2008) A Language and Environment for Statistical Computing. Vienna, Austria. Available at www.R-project.org

Reich D, Thangaraj K, Patterson N, et al. (2009) Reconstructing Indian population history. Nature 461:489-494.

Ross SE, Erickson RL, Gerin I, et al. (2002) Microarray analyses during adipogenesis: understanding the effects of Wnt signaling on adipogenesis and the roles of liver $\mathrm{X}$ receptor alpha in adipocyte metabolism. Mol Cell Biol 22:5989-5999.

Sanghera DK, Nath SK, Ortega L, et al. (2008) TCF7L2 polymorphisms are associated with type 2 diabetes in Khatri Sikhs from North India: genetic variation affects lipid levels. Ann Hum Genet 72:499-509. 
Schafer SA, Tschritter O, Machicao F, et al. (2007) Impaired glucagon-like peptide-1-induced insulin secretion in carriers of transcription factor 7-like 2 (TCF7L2) gene polymorphisms. Diabetologia 50:2443-2450.

Scott LJ, Mohlke KL, Bonnycastle LL, et al. (2007) A genomewide association study of type 2 diabetes in Finns detects multiple susceptibility variants. Science 316:1341-1345.

Sladek R, Rocheleau G, Rung J, et al. (2007) A genome-wide association study identifies novel risk loci for type 2 diabetes. Nature 445:881-885.

Vazquez G, Duval S, Jacobs DR, et al. (2007) Comparison of body mass index, waist circumference, and waist/hip ratio in predicting incident diabetes: a meta analysis. Epidemiol Rev 29: 115-128.
Zhang C, Qi L, Hunter DJ, et al. (2006) Variant of transcription factor 7-like 2 (TCF7L2) gene and the risk of type 2 diabetes in large cohorts of U.S. women and men. Diabetes 55:26452648.

Address correspondence to: Vipin Gupta, Ph.D. South Asia Network for Chronic Disease (SANCD) Public Health Foundation of India New Delhi 110016

India

E-mail: udaiig@gmail.com 